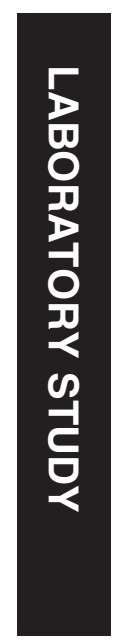

\title{
Melatonin prevents retinal oxidative stress and vascular changes in diabetic rats
}

${ }^{1}$ Department of

Ophthalmology, Faculty of Medicine, Kahramanmaraş Sütçüimam University, Kahramanmaraş, Turkey

${ }^{2}$ Department of Pharmacology, Faculty of Medicine, Kahramanmaraş Sütçüimam University, Kahramanmaraş, Turkey

${ }^{3}$ Department of Pathology, Faculty of Medicine, Kahramanmaraş Sütçüimam University, Kahramanmaraş, Turkey

${ }^{4}$ Department of Biochemistry, Faculty of Medicine, Kahramanmaraş Sütçüimam University, Kahramanmaraş, Turkey

Correspondence: G Özdemir, Kahramanmaraş Sütçüimam Üniversitesi, Tıp Fakültesi Göz Hastalıkları, Avşar Kampüsü 46100 Kahramanmaraş, Türkiye

Tel: +90 344280 3434;

Fax: +90 3442804052

E-mail: gozdemir@

hotmail.com or gozdemir@ ksu.edu.tr

Received: 28 August 2013 Accepted in revised form: 24 April 2014

Published online:

13 June 2014

\begin{abstract}
Purpose To evaluate the role of melatonin, an antioxidant agent, in diabetic oxidative stress and vascular damage.

Methods Diabetes was induced in 21 male Wistar rats by intraperitoneal (IP) administration of streptozotocin and then the rats were equally and randomly allocated to diabetic, melatonin, and vehicle groups. Seven healthy normal rats with similar features comprised the control group as the fourth group. All animals were followed for 12 weeks. The melatonin group received IP melatonin daily and the vehicle group received $2.5 \%$ ethanol IP at the last month. At the end of 12 weeks, the rats were killed and retinas were harvested. The retinas were investigated for the existence of hypoxiainducible factor 1- $\alpha$ (HIF-1 $\alpha$ ), vascular endothelial growth factor A (VEGF-A), and pigment epithelium-derived factor (PEDF) by ELISA. Retinal oxidative stress is quantitated by measuring nitrotyrosine and malondialdehyde levels. Retinal immunohistochemistry with antibody against CD31 antigen was carried out on retinal cross-sections. For statistics, ANOVA test was used for multiple comparisons.

Results Hyperglycemia increased retinal oxidation as measured through levels of nitrotyrosine and malondialdehyde. Diabetic retinas are also associated with abnormal vascular changes such as dilatation and deformation. HIF- $1 \alpha$, VEGF-A, and PEDF were all increased because of diabetic injury. Melatonin showed a potential beneficial effect on retinopathy in diabetic rats. It decreased retinal nitrotyrosine and malondialdehyde levels, showing an antioxidative support. The vasculomodulator cytokines are decreased accordingly by melatonin therapy. Melatonin normalized retinal vascular changes as well.
\end{abstract}

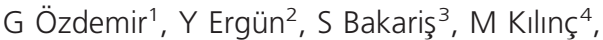
H Durdu ${ }^{3}$ and E Ganiyusufoğlu ${ }^{4}$

Conclusion Melatonin may show some advantage on diabetic vascular changes through decreasing oxidative stress and vessel-related cytokines.

Eye (2014) 28, 1020-1027; doi:10.1038/eye.2014.127; published online 13 June 2014

\section{Introduction}

Diabetic retinopathy is a common disorder and a leading cause of blindness worldwide. ${ }^{1}$

Although some aspects of the disease have been enlightened, most are yet under investigation.

Some evidence revealed that increased oxidative stress takes place in the progression of diabetic disorder. ${ }^{2}$ Activated oxygen species such as hydrogen peroxide, superoxide anion, and singlet oxygen are produced during the normal respiratory metabolism. Cells have protective counterbalancing systems against these free radicals. Nevertheless, in case radicals are overproduced, scavenger systems become overweighted and oxidative imbalance emerges. Diabetes is a known inducer of oxyradicals in the body and hyperglycemia is associated with oxidative stres through induction of several pathways. Nitric oxide (NO) may contribute to oxidative damage through nitrosylation of proteins in the existence of abundant oxygen radicals. Especially, retina is vulnerable to and the target for this kind of damage. ${ }^{3}$

Diabetes causes upregulation of angiogenesis modulating factors. Vascular endothelial growth factor (VEGF), hypoxia-inducible factor (HIF), and pigment epithelium-derived factor (PEDF) are produced reactively to tissue injury occurred during diabetic process. ${ }^{4}$ The diabetic microvasculopathy is strongly associated especially with VEGF. Vascular dilatation, microaneurysm, and other vascular abnormalities seen in diabetic retinopathy are primarily carried out by VEGF. Researches revealed that the disease can be limited by targeting these factors. ${ }^{5}$ 
Antioxidants have been reported to exert possible protective actions in diabetic retinopathy. ${ }^{6}$ A diurnal hormone, melatonin, is an oxyradical scavenger and stimulates endogenous antioxidant enzymes. Various investigators indicated a strong antioxidative action of melatonin in several diseases. ${ }^{7}$ In addition, an advantage on blood glucose levels was reported with melatonin use. ${ }^{8}$ On this background, we designed a study to elucidate the association between oxidative stress, vasculogenic mediators, and melatonin in type 1 diabetic rats induced by streptozotocin.

\section{Materials and methods}

\section{Animal care and the induction of diabetic retinopathy}

This study was funded by 'Scientific Research Project Committee of KSU'. The experimental protocol was approved before the study by 'The Ethics Committee for Animal Care and Use in Research and Education' and all animals received humane care and treatment in accordance with the ARVO Statement for the Use of Animals in Ophthalmic and Vision Research. The animals were housed in polypropylene cages inside a well-ventilated room. Each cage consisted of not more than three rats. They were maintained under standard laboratory conditions of temperature $22-24{ }^{\circ} \mathrm{C}$, relative humidity $60-70 \%$, and $12 \mathrm{~h}$ light-dark cycle. They were fed a standard commercial pellet diet and water ad libitum.

Experimental diabetes was induced in adult male Wistar rats of 12 weeks of age by a single intraperitoneal injection (IP) of $60 \mathrm{mg} / \mathrm{kg}$ streptozotocin. All rats were fasted for $24 \mathrm{~h}$ before diabetes was induced. Rats with a blood glucose level of $250 \mathrm{mg} / \mathrm{dl}$ and over $48 \mathrm{~h}$ after the injection were regarded diabetic and followed for 12 weeks.

Four groups, each of which contained 7 rats, were formed. In diabetic group, diabetic rats were followed without any drugs. In melatonin + diabetes group, diabetic rats were given IP melatonin (MP Biomedicals, Strasbourg, France) $10 \mathrm{mg} / \mathrm{kg}$ (dissolved in $2.5 \%$ ethanol) in $1 \mathrm{ml}$ daily for 4 weeks at the last month.

Vehicle + diabetes group consisted of diabetic animals that received only $1 \mathrm{ml}$ of $2.5 \%$ ethanol IP given last month. For comparison purposes, healthy male rats of the same age were recruited into the control group. Both diabetic and control group received $1 \mathrm{ml}$ physiological saline IP daily at the last month. All injections were given at $1700 \mathrm{~h}$. All animals were followed weekly with regard to the weight and blood sugar. At the end of the third month, all rats were killed with a cardiac puncture under ketamin $(50 \mathrm{mg} / \mathrm{kg}, \mathrm{IP})$ and xylazine $(3 \mathrm{mg} / \mathrm{kg}, \mathrm{IP})$ anesthesia.

\section{Retinal biochemical analysis}

The retinas from the left eyes were dissected right away after killing and kept refrigerated at $-70^{\circ} \mathrm{C}$ until biochemical analyses were done. For the assays, retina samples were placed in a cold phosphate-buffered saline (diluted ratio $1: 5 ; \mathrm{pH} 7.2$ ) and homogenized with a mechanic homogenizator as described by Masuzawa et al. ${ }^{10}$ Aliquots of homogenates were then centrifuged at $10000 \mathrm{~g}$ for $30 \mathrm{~min}$ and the supernatants were analyzed for the investigations. Enzyme-linked immunosorbent assay (ELISA) was performed for determinations of retinal VEGF, HIF- $1 \alpha$, PEDF, and nitrotyrosine. ELISA kit for PEDF, HIF-1- $\alpha$, and nitrotyrosine were obtained from Cusabio (Wuhan, Hubei, China) and for VEGF from eBioscience (San Diego, CA, USA). Results for VEGF, HIF- $1 \alpha$ and PEDF are expressed as picogram per milligrams of protein (pg/mg-prot). The result for nitrotyrosine is expressed as nanogram per milligram of protein (nmol/mg-prot).

The retina lipid peroxidation was assessed by thiobarbituric acid reactive substances (TBARS) measurement according to Ohkawa et al ${ }^{11}$ with modifications. The results were expressed in nanomoles per milligram protein (nmol/mg protein). Retinal protein was measured according to Lowry et al. ${ }^{12}$

\section{Retinal immunohistochemistry for CD31}

Panendothelial cell marker (CD31) was used to determine vascular changes as described elsewhere. ${ }^{9}$ Briefly, eyes were perfused with $4 \%$ paraformaldehyde intracardiacly and then eyes were enucleated. A corneal puncture was made and eyes were put into $4 \%$ paraformaldehyde for $48 \mathrm{~h}$. Immunohistochemical staining was performed on formalin-fixed, parafinembedded tissue by using the streptavidin-biotin method and 4-5 sections were obtained for evaluation from each eye. Starting from close vicinity to optic nerve, whole-tissue paraffin sections $(3-4 \mu \mathrm{m})$ were obtained parallel to the optic disc and the sections were deparaffinated in xylene and rehydrated through graded series of ethanol solutions. Tissue sections were subject to antigen retrieval by microwave treatment in $0.01 \mathrm{M}$ sodium citrate buffer ( $\mathrm{pH}$ 6.0) at $900 \mathrm{~W}$. Endogenous peroxidase activity was blocked by incubating the slides in $3 \%$ hydrogen peroxide in distilled water for $5 \mathrm{~min}$. Primary antibody incubations were: anti-CD-31 (mouse monoclonal, Santa Cruz Biotechnology, Santa Cruz, CA, USA) incubated for $1 \mathrm{~h}$ in a humidity chamber at room temperature. Slides were counterstained with Mayer's hematoxylin. The isotype controls were also done for immunohistochemistry. The immunstained specimens were evaluated by using light microscopes and pictures were taken with an digital camera attached to the microscope. 


\section{Blood biochemical analyses}

Blood sample $(5 \mathrm{ml})$ was obtained intracardiac for blood biochemistry. Blood biochemical parameters including complete blood count, glucose, HbA1c, blood urea nitrogen, creatinine, and albumin levels were measured. Hematological parameters ( $\mathrm{Hb}, \mathrm{Hct}$, and WBC count) were measured from complete blood by using an electronic blood count instrument (Advia 2120i, Siemens, Germany) within $24 \mathrm{~h}$. HbA1c levels were measured with an automated analyzer (high-performance liquid chromatography; Bio-Rad D10, Hercules, CA, USA). Blood biochemical parameters (glucose, blood urea nitrogen, creatinin, albumin) were analyzed with an automated biochemical analyzer (Dade RXL, Dimension, Deerfield, IL, USA). All parameters were calculated according to own SD.

Data were entered into an SPSS database (version 16, SPSS Inc., Chicago IL, USA). To compare groups, one-way analysis of variance (one-way ANOVA) was used. The post hoc multiple comparisons among the groups were done with Tukey's honestly significant differences test after the group variances were shown to be equal by Levene's test. The data were expressed as mean \pm SD (95\% confidence interval). A $P$-value of $<0.05$ was considered statistically significant.

\section{Results}

At the time of killing, the mean weights of the rats were $220.42 \pm 23.17 \mathrm{~g}(200-235)$ in the melatonin group, $190.22 \pm 20.98 \mathrm{~g}(170-220)$ in the diabetic group, $170.75 \pm 28.17 \mathrm{~g}(148-207)$ in the vehicle group, and $287.00 \pm 21 \mathrm{~g}(266-309)$ in the control group. Both diabetic and melatonin groups had lower body weights compared with controls $(P<0.05)$.

IP streptozotocin caused a significant rise in blood sugar. The blood sugar was high in all diabetic animals including melatonin group $(P<0.05)$. Similarly, these groups also had higher HbA1c levels $(P<0.05)$. Glucose level was $662 \mathrm{mg} / \mathrm{dl}$ in the diabetic group, $611 \mathrm{mg} / \mathrm{dl}$ in the melatonin + diabetic group, $603 \mathrm{mg} / \mathrm{dl}$ in the vehicle + diabetic group, and $107 \mathrm{mg} / \mathrm{dl}$ in the control group The HbA1c level was $13.63 \% \pm 10$ in the diabetic group, $11.92 \% \pm 1.72$ in the melatonin + diabetic group, $12.4 \% \pm 4.2$ in the vehicle + diabetic group, and $7.35 \% \pm 3.3$ in the control group. There were no significant differences with regard to blood glucose and $\mathrm{HbA1c}$ levels between diabetic animals.

\section{Retinal oxidation}

Diabetes increased retinal nitrotyrosine by $80 \%$ and malondialdehyde levels twice as compared with controls $(P<0.05)$. The retinal oxidation responded favorably to melatonin treatment $(P<0.05$; Figures 1 and 2$)$. Vehicle treatment did not show any benefit $(P>0.05)$, meaning that the advantage is coming from totally melatonin administration.

\section{Retinal angiomodulator cytokines}

There was a dramatic increase in VEGF and PEDF levels of diabetic animals (Figure 3). VEGF showed an approximately triple increase $(P<0.001)$ in the diabetic group as compared with the control group. HIF- $1 \alpha$ also showed a similar increase in the diabetes group $(P<0.05$; Figure 4$)$. Melatonin had a substantial effect

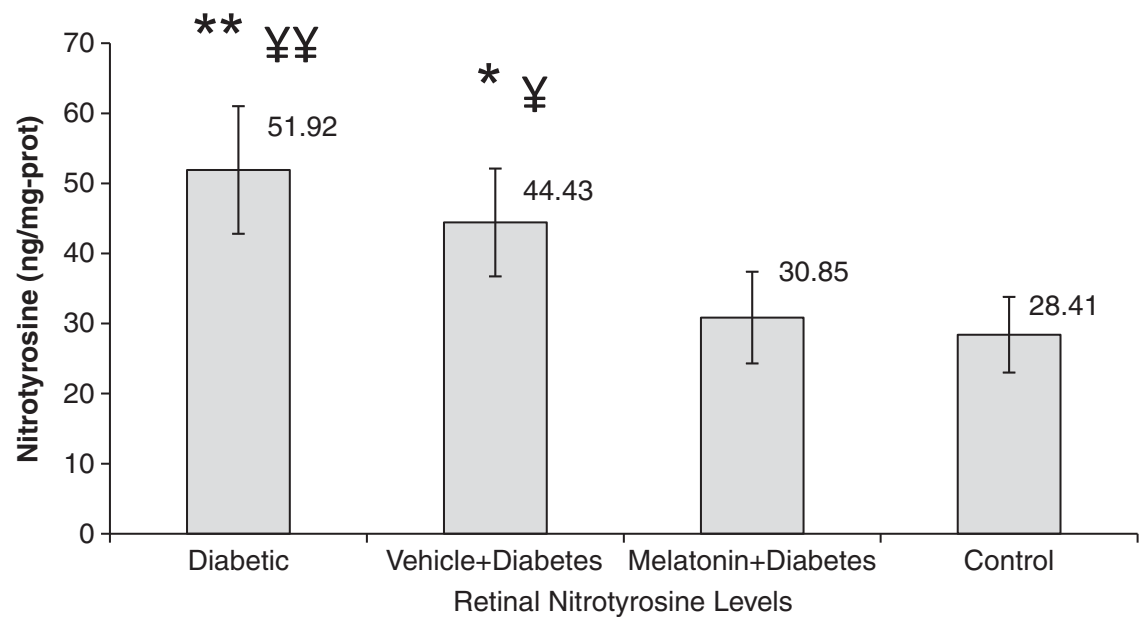

Figure 1 Retinal nitrotyrosine levels between the groups. Briefly, aliquots of homogenates were centrifuged and the supernatants were analyzed by ELISA method for retinal nitrotyrosine. Results were expressed in $\mathrm{ng} / \mathrm{mg}$ protein. Error bars represent SD. Data are given as mean $\pm \mathrm{SD}$. ${ }^{* *} P<0.001$ compared with control group, ${ }^{\text {¥¥ }} P=0.006$ compared with melatonin + diabetes group, ${ }^{*} P=0.033$ compared with control group, ${ }^{\sharp} P=0.046$ compared with melatonin + diabetes group. 


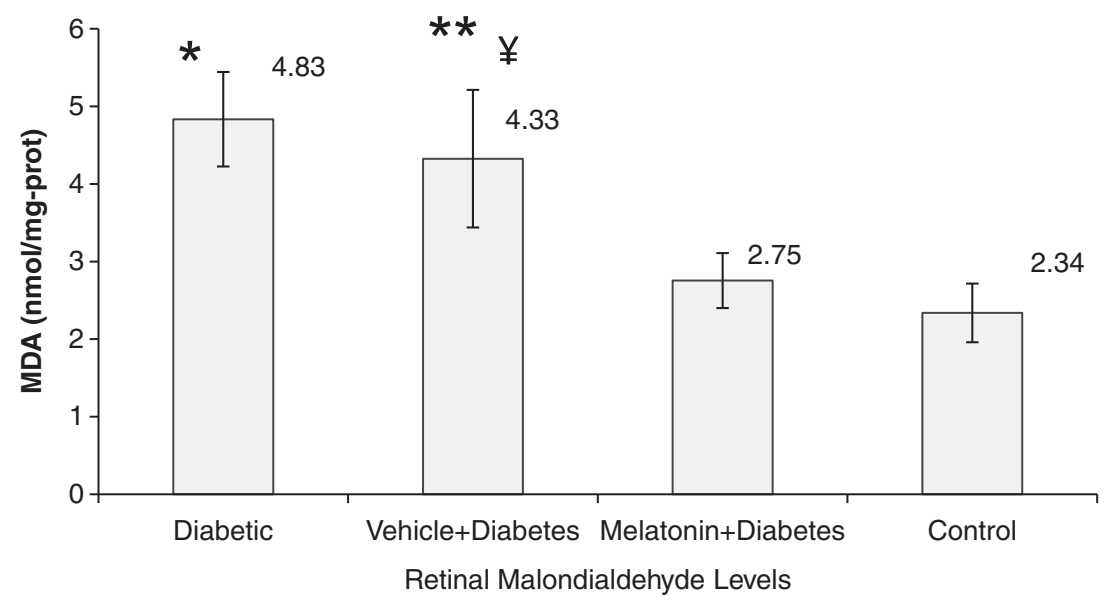

Figure 2 Retinal lipid peroxidation levels in diabetic and control rats. The retina lipid peroxidation (total MDA) were determined according to Ohkawa et $\mathrm{al}^{11}$ with modifications. Results were expressed in $\mathrm{nmol} / \mathrm{mg}$ protein. Error bars represent SD. Data are given as mean \pm SD. ${ }^{*} P<0.001$ compared with control and melatonin + diabetes groups, ${ }^{* *} P<0.001$ compared with control group, ${ }^{*} P<0.01$ compared with melatonin + diabetes group.

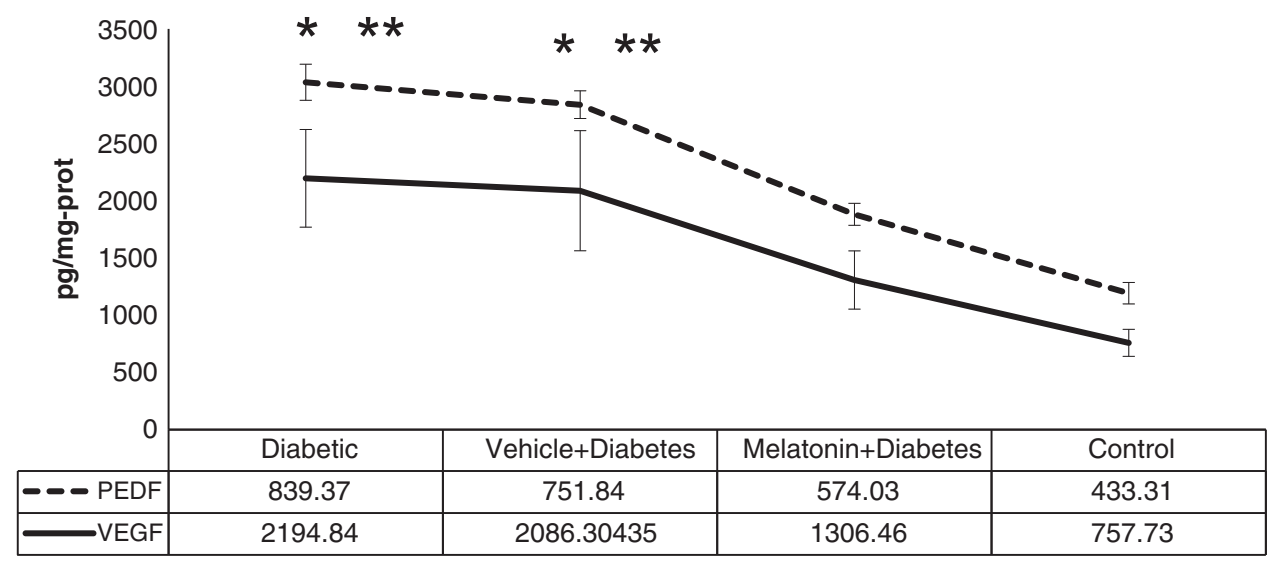

Retinal VEGF and PEDF levels

Figure 3 The mean VEGF and PEDF levels between the groups. Briefly, aliquots of homogenates were centrifuged and the supernatants were analyzed by ELISA method. Results were expressed in pg/mg protein. Error bars represent SD. Data are given as mean $\pm \mathrm{SD}$. ${ }^{*} P<0.001$ compared with control group, ${ }^{* *} P<0.05$, compared with melatonin + diabetes group.

in decreasing these cytokines in the treatment group $(P<0.05)$. It decreased HIF1- $\alpha$ and PEDF to normal levels and prohibited the increment in VEGF considerably. Vehicle administration had no effect on those cytokines $(P>0.05)$.

\section{Retinal immunohistochemistry}

Immunohistochemistry revealed vascular engorgement and dilatation in diabetic animals (Figure 5). CD31 staining was more denser in diabetic retinas. The increased luminal space is also shown. With melatonin use, vascular derangement disappeared and vascular distortion was lost. Melatonin treatment resulted in a better retinal outcome with respect to the vascular structure.

\section{Discussion}

Streptozotocin is an antibiotic commonly used in pancreatic islet cell carcinoma. When uptaken, it causes rapid degeneration and necrosis of these cell, resulting in a condition similar to type 1 diabetes mellitus. In our study, animals developing diabetes demonstrated failure in weight gain compared with healthy counterparts $(P<0.05)$

In our study, the retinal images showed vascular engorgement in the diabetic group. We also confirmed this morphological changes biochemically by measuring tissue VEGF levels. Abnormal vascular changes such as dilatation, microaneurysm, and tortuosity are prominent features of diabetic retinopathy. Preponderant CD-31 staining with marked vascular dilatations were reported 


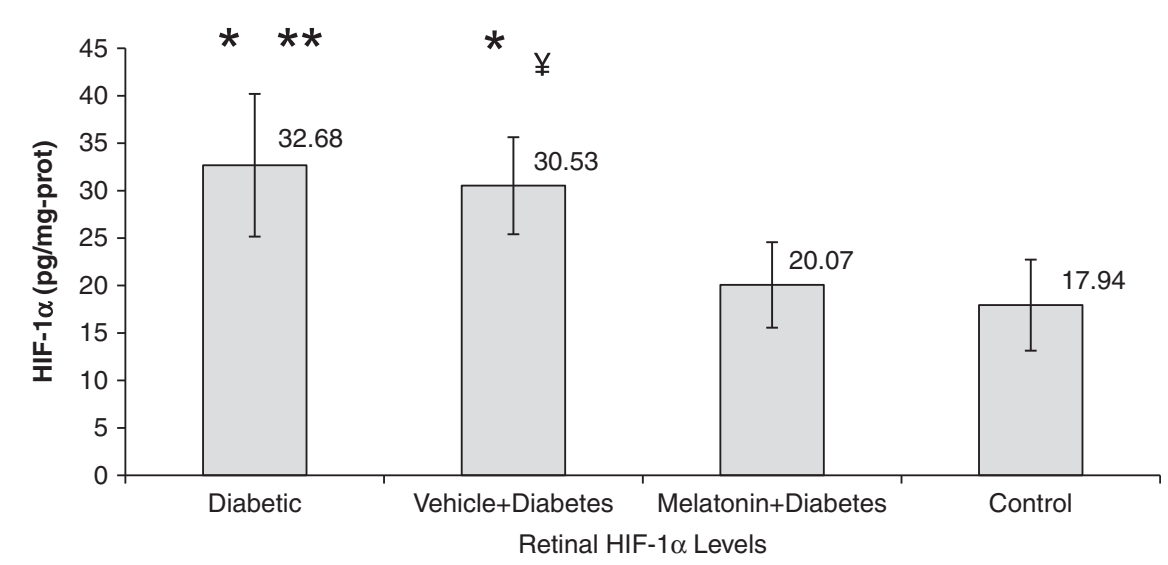

Figure 4 Retinal HIF- $1 \alpha$ levels between the groups. Briefly, aliquots of homogenates were centrifuged and the supernatants were analyzed by ELISA method. Results were expressed in $\mathrm{pg} / \mathrm{mg}$ protein. Error bars represent SD. Data are given as mean $\pm \mathrm{SD}$. ${ }^{*} P<0.01$ compared with control group, ${ }^{* *} P=0.031$ compared with melatonin + diabetes group, ${ }^{*} P=0.042$ compared with melatonin + diabetes group.

in diabetic patients elsewhere, mainly created by high levels of VEGF.9,13 This vessel active cytokine plays an important role in the development of diabetic complications such as edema and hemorrhages. Although we did not measure retinal edema marker such as PAL-E antigen or fibrinogen, they are mostly to accompany such kind of vascular deformation. ${ }^{13}$

Melatonin supplementation was successful to reduce diabetic microvascular deterioration in this study. Subcutaneous implantation of melatonin therapy prevented retinal VEGF staining in diabetic rats. ${ }^{7}$ Reduced VEGF secretion by melatonin may be a substantial factor in ameliorating vascular deformation. How melatonin decreases VEGF may be a concern of future investigations.

As showed elsewhere, ${ }^{14}$ diabetic retinopathy was associated with oxidative and nitrosative stress in our experiment. Diabetes increases NO via upregulation of iNOS, mostly through a mechanism involving Mueller cell function. ${ }^{3}$ In the presence of superoxide, NO forms peroxynitrite and leads to lipid peroxidation and DNA damage. Another study established the elevated nitrotyrosine levels in nerve fiber layer and endothelial cells of diabetic retina. ${ }^{15}$ These evidence suggest a pivotal role of NO in diabetic retinopathy. ${ }^{16}$ Peroxynitrite reacts with protein through tyrosine and forms nitrotyrosine that is used as a marker of nitration in the tissue. Our experiment revealed a high nitrotyrosine level in diabetic group, confirming nitrative damage.

Malondialdehyde (MDA) is one of several lowmolecular-weight end products formed via the decomposition of lipid peroxidation. Malondialdehyde and other thiobarbituric acid reactive substances (TBARS) condense with thiobarbituric acid to give a fluorescent red derivative that can be assayed spectrophotometrically. TBARS are widely known indicator of oxygen radicals attack. ${ }^{10}$ However, some other agents such as aldehydes and ketones also react with thiobarbituric acid that may create a possible confounding factor. To eliminate the limitation of MDATBARS measurement, we compared our results with control group and verified for other possible TBAreactive substances between the group comparisons. In our experiment, diabetic group had higher MDA levels compared with control. Hyperglycemia oxidized retina and increased MDA content more than twofold. Studies showed that hyperglycemia causes oxidation via induction of several pathways, including protein kinase C, sorbitol, and phoshoinositide 3-kinase pathways. ${ }^{17,18}$

Free radicals impair cellular membranes and may disrupt endothelial cell integrity, creating both ischemic and edematous areas. ${ }^{19}$ Oxidative stress may be crucial in diabetic retinopathy and clinical implications of diabetes may be carried out by reactive oxygen species. ${ }^{20}$

Melatonin (5-methoxy-N-acetyltryptamine) is a neurohormone mainly secreted by pineal gland. Apart from pineal gland, it is also synthesized in the retina. ${ }^{21}$ In contrary to the pineal melatonin, which acts as a systemic hormone, retinal melatonin exerts its effects as a paracrine neuromodulator. Melatonin is secreted from photoreceptor cells in the retina and activates dark induced changes. Retinal diurnal rhytmicity are heavily related to the melatonin and light interaction. During the night, retinal melatonin synthesis increases, and during the daytime vice versa occurs. Once secreted, it can easily diffuse throughout the retina because of its lipophilic and hydrophilic nature and exerts its functons through MT1 and MT2 receptors, both of which exist within the eye. ${ }^{21-23}$

Melatonin can be dissolved in dimethylsulfoxide or ethanol succesfully. Previous studies showed that 

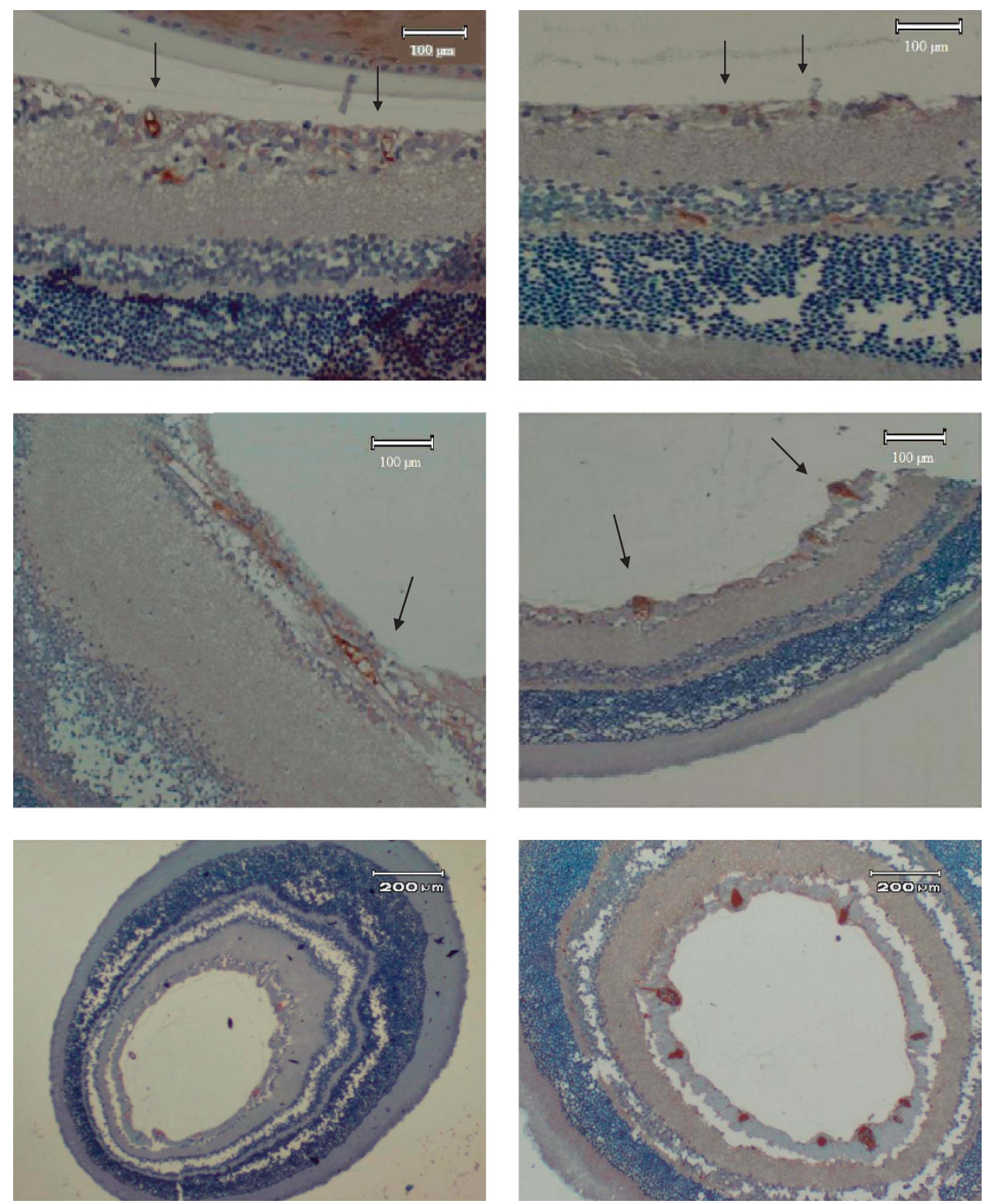

Figure 5 Immunoperoxidase staining of retinal sections of rats with anti-CD31. (Top left) Normal rat retina (control group) showed several small vessels stained pale (arrows) (magnification: $10 \times 10$ ). (Top right) Diabetic rat retina treated with melatonin (melatonin + diabetes group). Diffuse small pale stainings are observed (arrows) (magnification: $10 \times 10$ ). (Middle left) Diabetic rat retina (diabetic group). A diffuse and intense staining is noted (magnification: $10 \times 10$ ). (Middle right) Diabetic retina treated with vehicle (vehicle + diabetes group) instead of melatonin. Abnormal vessels with intense staining (arrows) are seen (magnification: $10 \times 10$ ). Large diameter vessels are also seen. (Lower left) Normal rat retina (control group) with lower magnification $(4 \times 10)$. Small pale pinkstained vessels are seen especially in the nerve fiber layer. (Lower right) Diabetic rat retina (diabetic group) with lower magnification $(4 \times 10)$. Intensely stained and large size vessels are prominent in the nerve fiber layer of the retina.

melatonin membrane receptors were distributed throughout the retina ${ }^{23}$ and melatonin administered systemically could reach to the eye, increasing retinal methoxyindole levels.?

We found that melatonin decreased nitrotyrosine in retinal homogenates obtained from diabetic rats, whereas vehicle administration had no effect on glucose-induced damage. It is reported that melatonin suppresses the activity of NO synthase. ${ }^{7}$ Accordingly, the limited NO production may prevent the development of nitrative stres under hyperoxic conditions, as shown herein.

Another contribution by melatonin is its free radical scavenging property. ${ }^{24}$ Melatonin is a known antioxidant and shows antioxidant activity in the retina. ${ }^{25}$ Melatonin can remove free radicals directly from the environment 
effectively and forms stable irreversible metabolites. Therefore, it has been referred to as a terminal (or suicidal) antioxidant. This aspect might be related to the diminished damage in this study.

A posivite impact on the activity of antioxidant enzymes by melatonin is also possible inasmuch as superoxide dismutase, glutathione peroxidase, and calatalase levels are favored with melatonin therapy. 7,24 These enzymes take active part in the elimination of rambling radicals through redox reactions, yielding water and molecular oxygen. Although we did not measure retinal antioxidant enzyme levels, our possible results may be linked to this situation.

Diabetic rat retinas showed increased levels of HIF-1 $\alpha$ and VEGF levels in this experiment, as reported elsewhere. ${ }^{26}$ Diabetic retinopathy is associated with an increased level of VEGF even before the development of acellular capillaries. ${ }^{2,4,27}$ Hypoxia blocks HIF-1 $\alpha$ degradation and increases its accumulation. Diabetic hypoxia may be a cause for increased levels of HIF- $1 \alpha$ that in turn stimulates VEGF synthesis. Furthermore, VEGF, a cytoprotective factor, can be activated upon various insults including hyperglycemia and oxidative stress may be a key factor for stimulation. From another point of view, it has been reported that raised level of VEGF may be a precipitating event in diabetic retinopathy. ${ }^{12}$ When the milieu is stressed oxidatively, it may activate pathological cascades paradoxically and increase superoxide radicals. ${ }^{14,28}$ Obviously, this issue requires further consideration. In our study melatonin administration prevented the increase in HIF- $1 \alpha$ and VEGF levels. A direct suppressive effect of melatonin on vascular endothelial growth factor is reported at Mueller cell level. ${ }^{23}$ Cessation of oxidative damage through melatonin treatment may have led to the decrease in these cytokines as well. Melatonin's effect on angiomodulator agents may be a promisory approach in microvasculopathies.

PEDF is a glycoprotein that was firstly identified from fetal human retinal pigment epithelial cell culture. ${ }^{29}$ It is widely expressed in the retina and has potent prosurvival and antioxidant features. We detected higher levels of PEDF in the diabetic group as compared with the control animals. Although several authors found that hyperglycemia depressed the level of PEDF in cell cultures, ${ }^{30}$ controversy still exists. Elevated levels of PEDF in diabetic rats were demonstrated by some authors in accordance with our results. ${ }^{31}$ It may be argued that PEDF could be increased secondarily to protect the retina in response to diabetic impairment. With a chronic ongoing damage, PEDF depletion may be a possibility. ${ }^{17,28}$ Melatonin in our study also prohibited the raised level of PEDF. PEDF may have been reduced because of diminished oxidative stress by melatonin.
In addition, a direct inhibitory effect with melatonin on PEDF synthesis should be investigated as well.

In our study, hyperglycemia and HbA1c levels between diabetic and melatonin groups were comparable and very high compared with the controls $(P<0.05)$. We detected that glucose was increased more than fivefold and $\mathrm{HbA1c}$ roughly twofold in diabetic animals. Although melatonin is reported to have decreased blood sugar, ${ }^{8}$ this is not the case in our study. Other researchers reported no effect of melatonin on blood glucose levels like us. ${ }^{7,24}$ These conflicting results may be related to the strains and the genetic variations of the animals. Melatonin treatment did not cause any disorder on blood biochemical findings.

As a result, we were successful in reducing diabetic retinal damage with melatonin supplementation. Being an ideal antioxidant because of its unique features, melatonin may have the potential for therapeutic applications. Further analyses are required to elucidate the effect of melatonin on diabetic complications.

\section{Summary}

What was known before

- Diabetic retinopathy is associated with increased oxidative stress and vasogenic hormones.

- Vascular complications also play an important role in this disease.

What this study adds

- Melatonin may reduce diabetic oxidative stress and vasogenic hormones including vascular endothelial growth factor.

- It may also show beneficial vascular changes.

\section{Conflict of interest}

The authors declare no conflict of interest.

\section{Acknowledgements}

This study was supported by Kahramanmaras Sutcuimam University Scientific Research Project Committee (project number: 2011/6-2M).

\section{References}

1 Cai J, Boulton M. The pathogenesis of diabetic retinopathy: old concepts and new questions. Eye 2002; 16: 242-260.

2 Antonetti DA, Barber AJ, Bronson SK, Freeman WM, Gardner TW, Jefferson LS et al. Diabetic retinopathy. Seeing beyond glucose-induced microvascular disease. Diabetes 2006; 55: 2401-2411.

3 Du Y, Smith MA, Miller CM, Kern TS. Diabetes induced nitrative stress in the retina, and correction by aminoguanidine. J Neurochem 2002; 80: 771-779. 
4 Curtis TM, Gardiner TA, Stitt AW. Microvascular lesions of diabetic retinopathy: clues towards understanding pathogenesis. Eye 2009; 23: 1496-1508.

5 Frank RN. Diabetic retinopathy. N Eng J Med 2004; 350: 48-58.

6 Kowluru RA, Menon B, Gierhart DL. Beneficial effect of zeaxanthin on retinal metabolic abnormalities in diabetic rats. Invest Ophthalmol Vis Sci 2008; 49: 1645-1651.

7 Salido EM, Bordone M, De Laurentiis A, Chianelli M, Keller Sarmiento MI, Dorfman D et al. Therapeutic efficacy of melatonin in reducing retinal damage in an experimental model of early type 2 diabetes in rats. J Pineal Res 2013; 54: 179-189.

8 Anwar MM, Meki AR. Oxidative stress in streptozotocin induced diabetic rats: effects of garlic oil and melatonin. Comp Biochem Physiol A 2003; 135: 539-547.

9 Hofman P, van Blijswijk BC, Gaillard PJ, GFJM Vrensen, Schlingemann RO. Endothelial cell hypertrophy induced by vascular endothelial growth factor in the retina. New insights into the pathogenesis of capillary nonperfusion. Arch Ophthalmol 2001; 119: 861-866.

10 Masuzawa K, Jesmin S, Maeda S, Zaedi S, Shimojo N, Miyauchi Tet al. Effect of endothelin dual receptor antagonist on VEGF levels in streptozotocin-induced diabetic rat retina. Exp Biol Med (Maywood) 2006; 23: 1090-1094.

11 Ohkawa H, Ohishi N, Yagi K. Assay for lipid peroxides in animal tissues by thiobarbituric acid reaction. Anal Biochem 1979; 95: 351-358.

12 Lowry OH, Rosebrough NJ, Farr AL, Randaii RJ. Protein measurement with the Folin phenol reagent. J Biol Chem 1951; 193: 265-275.

13 Schlingemann RO, Hofman P, GFJM Vrensen, Blaauwgeers HGT. Increased expression of endothelial antigen PAL-E in human diabetic retinopathy correlates with microvascular leakage. Diabetologia 1999; 42: 596-602.

14 El-Remessy AB, Behzadian MA, Abou-Mohamed G, Franklin T, Caldwell RW, Caldwell RB. Experimental diabetes causes breakdown of the blood-retina barrier by a mechanism involving tyrosine nitration and increases in expression of vascular endothelial growth factor and urokinase plasminogen activator receptor. Am J Pathol 2003; 162: 1995-2004.

15 Ellis EA, Guberski DL, Hutson B, Grant MB. Time course of NADH Oxidase, inducible nitric oxide synthase and peroxynitrite in diabetic retinopathy in the BBZ/WOR Rat. Nitric Oxide 2002; 6: 295-304.

16 Yılmaz G, Esser P, Kociek N, Aydın P, Heimann K. Elevated vitreous nitric oxide levels in patients with proliferative diabetic retinopathy. Am J Ophthalmol 2000; 130: 87-90.
17 Witmer AN, GFJM Vrensen, van Noorden CJF, Schlingemann RO. Vascular endothelial growth factors and angiogenesis in eye disease. Prog Retin Eye Res 2003; 22: 1-29.

18 Arnal E, Miranda M, Johnsen-Soriano S. Beneficial effect of docosahexanoic acid and lutein on retinal structural, metabolic, and functional abnormalities in diabetic rats. Curr Eye Res 2009; 34: 928-938.

19 Hardy P, Dumont I, Bhattacharya M, Hou X, Lachapelle P, Varma DR et al. Oxidants, nitric oxide and prostanoids in the developing ocular vasculature: a basis for ischemic retinopathy. Cardiovasc Res 2000; 47: 489-509.

20 Hammes HP, Feng Y, Pfister F, Brownlee M. Diabetic retinopathy: targeting vasoregression. Diabetes 2011; 60: 9-16.

21 Huang H, Wang Z, Weng SJ, Sun XH, Yang XL. Neuromodulatory role of melatonin in retinal information processing. Prog Retin Eye Res 2013; 32: 64-87.

22 Tosini G, Iuvone PM, Boatright JH. Is the melatonin receptor type 1 involved in the pathogenesis of glaucoma. J Glaucoma 2013; 22: S49-S50.

23 Jiang T, Chang Q, Zhao Z, Yan S, Wang L, Cai J et al. Melatonin mediated cytoprotection against hyperglycemic injury in Müller cells. PLoS One 2012; 7: e50661.

24 Maritim AC, Moore BH, Sanders RA, Watkins JB III. Effects of melatonin on oxidative stres in stretozotocin induced diabetic rats. Int J Toxicol 1999; 18: 161-166.

25 Tosini G, Baba K, Hwang CK, Iuvone PM. Melatonin an underappreciated player in retinal physiology and pathophysiology. Exp Eye Res 2012; 103: 82-89.

26 Arjamaa O, Nikinmaa M. Oxygen-dependent diseases in the retina: role of hypoxia-inducible factor. Exp Eye Res 2006; 83: 473-483.

27 Stefansson E. Ocular oxygenation and the treatment of diabetic retinopathy. Surv Ophthalmol 2006; 51: 364-380.

28 Penn JS, Madan A, Caldwell RB, Bartoli M, Caldwell RW, Hartnett ME. Vascular endothelial growth factor in eye disease. Prog Retin Eye Res 2008; 27: 331-371.

29 Boehm BO, Lang G, Volpert O, Jehle PM, Kurkhaus A, Rosinger $\mathrm{S}$ et al. Low content of the natural ocular anti-angiogenic agent pigment epithelium-derived factor (PEDF) in aqueous humor predicts progression of diabetic retinopathy. Diabetologia 2003; 46: 394-400.

30 Wang B, Atherton P, Patel R, Manning G, Donnelly R. Antiangiogenic effects and transcriptional regulation of PEDF in diabetic retinopathy. Microvasc Res 2010; 80: 31-36.

31 Matsuoka M, Ogata N, Minamino K, Higuchi A,

Matsumura M. High levels of pigment epithelium-derived factor in the retina of a rat model of type 2 diabetes. Exp Eye Res 2006; 82: 172-178. 